\title{
Preparação e Avaliação de Novos Nanosistemas Terapêuticos de Liberação Prolongada de Bentonita - Aciclovir
}

\author{
Flávia Almada do Carmo, Lúcio Mendes Cabral, Camila Braga \\ Dornelas e Michele Villardi
}

Fármacos antivirais, em especial aqueles que apresentam baixa estabilidade e destinados ao uso tópico, podem ter sua eficácia farmacológica modulada pelo controle de sua liberação a partir da forma farmacêutica na qual este é veiculado. Como possibilidades para sua formulação, considera-se como mais evidente a preparação de sistemas de liberação modificada de fármacos. A nanotecnologia, em especial, excipientes a base de nanocompósitos fármaco - "silicato lamelar", figura entre as melhores alternativas tecnológicas disponíveis para esta finalidade. Neste trabalho, tomou-se como objetivo principal o desenvolvimento de novos nanomateriais e nanocompósitos como sistemas carreadores de fármacos, focando-se, em ambos os casos, na otimização da liberação do aciclovir de forma a possibilitar seu uso como um novo sistema de liberação modificada, tanto de uso oral como de uso tópico.

Palavras-chave: liberação modificada; nanocompósito; nanomaterial; aciclovir.

For antiviral drugs, mainly those that present low stability and intended for topical use, their pharmacological effectiveness can be modulated through release control from the pharmaceutical delivery system in which it is formulated. There are some possibilities, but the preparation of modified release systems is more evident for this. Nanotechnology, with pharmaceutical active compound - silicate based, particularly, represents the best technological alternative available for this purpose.The aim of this work was to develop new nanomaterials and nanocomposites systems as drugs carriers, focusing, in both cases, the optimization of the acyclovir release to allow its use as a new system of modified release.

Keywords: modified release; nanocomposite; nanomaterial; acyclovir. 


\section{Introdução}

$\mathrm{Na}$ busca de novas alternativas terapêuticas para combater patologias que assolam a humanidade, novos fármacos têm surgido e outros ainda estão em pesquisa intensa dentro dos laboratórios das maiores indústrias farmacêuticas de todo o mundo ${ }^{1-3}$. Muitos destes novos fármacos, apesar de promissores em termos de sua atividade farmacológica, perdem em eficiência e aceitação visto sua baixa especificidade em relação ao alvo terapêutico, ou, ainda, sua biodisponibilidade reduzida. Ao mesmo tempo, esta mesma abordagem pode ser utilizada para "rejuvenescer" fármacos de uso consagrado em terapia, como o aciclovir, um anti-retroviral utilizado com sucesso em terapêutica desde $1977^{4-6}$.

Este fármaco apresenta, no entanto, as mesmas restrições da maioria dos quimioterápicos ${ }^{7}$, somando-se a estas sua baixa estabilidade, o que torna ainda mais problemática a sua formulação, em especial para uso tópico. A modulação de sua absorção tem sido estudada, sendo pouco explorado, todavia, o controle de sua liberação ${ }^{8-9}$. Este é um conceito específico enquadrado em um contexto mais amplo, o de liberação controlada de fármacos ${ }^{10-11}$. Para tal, um recurso evidente pode ser visto com o uso da nanotecnologia, podendo-se avaliar o desenvolvimento de sistemas de liberação de fármacos com base em silicatos lamelares, em especial a bentonita sódica e seus derivados organofílicos, como forma de modular a liberação deste ativo ${ }^{12}$.

\section{Materiais e Métodos}

Celulose microcristalina PH 102 (Avicel - FMC); aciclovir (Farmex - México), estearato de magnésio (Mallinkrodt); bentonita sódica, $95 \mathrm{meq} / 100 \mathrm{~g}$ de capacidade de troca catiônica (CEC) (Akros-Chemical); viscogel B8 ${ }^{\circledR}$ (Bentec). $\mathrm{HCl}$ (Tedia), apresentavam-se em grau analítico. Água MilliQ ${ }^{\circledR}$ foi utilizada para o preparo das soluções. O pacote de softwear STATISTICA (Stat Soft Company USA) foi utilizado nas análises estatísticas.

\section{REAÇÕES DE INTERCALAÇÃO FÁRMACO - SILICATOS LAMELARES}

Foram realizados experimentos de intercalação aciclovir - bentonita sódica e aciclovir - argila organofílica, variando-se, uma a um, temperatura (ambiente e sob refluxo), concentração de aciclovir (66meq, $88 \mathrm{meq}$, $150 \mathrm{meq}, 444 \mathrm{meq}$ e $888 \mathrm{meq} / 100 \mathrm{~g}$ de argila) e tempo (30min, 12h, 24h, 48h e 72h), empregando-se $\mathrm{HCl} 0,1 \mathrm{~N}$ como solvente. Como argila organofílica, foi utilizado viscogel $B 8 \AA$, resultante da inserção de octadecilamina na estrutura da bentonita sódica. A separação do material intercalado e não intercalado foi feita através de centrifugação (Sorvall RC2B), a 4000rpm por 1 hora. $\mathrm{O}$ rendimento foi estimado pela quantificação indireta da quantidade do fármaco presente no sobrenadante através de espectrofotometria de ultravioleta-visível (UV-Vis) (Biospectro), baseando-se nos valores da curva de calibração, previamente elaborada com aciclovir SQR em álcool etílico no comprimento de onda de $270 \mathrm{~nm}$, segundo compêndio oficial (USP 31).

Os materiais resultantes foram caracterizados por difração de raios X (DRX), em difratômetro Rigaku, modelo Miniflex, e espectrometria de infravermelho, em espectrofotômetro de infravermelho com transformada de Fourier, FTIR-8300, Shimadzu, de forma a se evidenciar a inserção do fármaco no espaço interlamelar dos silicatos lamelares estudados.

\section{PREPARAÇÃO DOS COMPRIMIDOS DE ACICLOVIR}

Foram produzidos três lotes de comprimidos, em compressora excêntrica, Piccola, por compressão direta (Tabela 1) de aciclovir intercalado com bentonita sódica, e um lote utilizando uma mistura física de aciclovir e bentonita sódica, nas mesmas proporções utilizadas no teste de interação (66meq/100g argila). Com os comprimidos preparados foram realizados testes de dissolução (dissolutor Nova Ética), para sistemas de liberação modificada, e dureza (durômetro Scheleuniger), segundo especificações farmacopeicas (USP31). O mesmo procedimento foi conduzido com os comprimidos obtidos com aciclovir - viscogel $\mathrm{B} 8^{\circledR}$.

Tabela 1: Formulação-base dos comprimidos de aciclovir

\begin{tabular}{|c|c|}
\hline Componente & Quantidade \\
\hline $\begin{array}{c}\text { Complexo Aciclovir } \\
+ \text { argila }\end{array}$ & qsp 100mg de aciclovir \\
\hline Celulose microcristalina & $20 \%$ \\
\hline Estearato de magnésio & $1,5 \%$ \\
\cline { 2 - 2 } & $500 \mathrm{mg}$ \\
\hline
\end{tabular}




\section{Resultados e Discussão}

\section{REAÇÕES DE INTERCALAÇÃO FÁRMACO - ARGILA SÓDICA}

O parâmetro temperatura foi estudado a fim de avaliar sua influência sobre o processo de intercalação. A condição de elevação da temperatura (de $30^{\circ} \mathrm{C}-$ temperatura ambiente para refluxo, $110^{\circ} \mathrm{C}$ ), objetivou um maior fornecimento de energia, de forma a se buscar um aumento no rendimento do processo. Os valores de rendimento são apresentados pela Tabela 2, a qual não evidencia diferença estatística entre as condições de temperatura testadas, ou seja, o aumento de temperatura para este processo não mostra qualquer vantagem. Todos os experimentos foram realizados em triplicata.

Após a avaliação da temperatura, foi proposto o estudo da influência da concentração de aciclovir no processo, variando-se, para isso, sua quantidade em massa, de modo a obter: 66meq, $88 \mathrm{meq}, 150 \mathrm{meq}, 444 \mathrm{meq} \mathrm{e} 888 \mathrm{meq} /$ $100 \mathrm{~g}$ de argila, conservando-se a condição previamente descrita como ótima (temperatura ambiente).

Segundo os resultados apresentados pela Tabela 3, a concentração considerada ótima foi de $444 \mathrm{meq} / 100 \mathrm{~g}$ de argila sódica, porque, além de ser a que resultou em uma maior massa da aciclovir fixada na argila em comparação as menores concentrações utilizadas, apresentou a maior reprodutibilidade de resultados do que os observados com o uso de $888 \mathrm{meq} / 100 \mathrm{~g}$ de argila.

Foram, ainda, realizadas reações de intercalação entre argila sódica e aciclovir utilizando-se os seguintes tempos de reação: 30min (Figura 1), 12h, 24h, 48h e 72h. Os materiais obtidos foram analisados por difração de raios X (Figura 2), onde não foram observadas diferenças significativas quanto ao espaçamento basal nos diferentes tempos considerados.

\section{REAÇÕES DE INTERCALAÇÃO FÁRMACO - ARGILA ORGANOFÍLICA}

Com relação ao ensaio de intercalação do aciclovir com bentonita organofílica, este seguiu as condições previamente tidas como ótimas de aciclovir e bentonita sódica, ou: a temperatura ambiente, com 444meq/ aciclovir/ 100g de argila em 30 minutos de reação.
Tabela 2: Rendimento da intercalação e quantidade de aciclovir intercalado em relação à temperatura

\begin{tabular}{|c|c|c|c|}
\hline Temperatura & $\begin{array}{c}\text { Abs. } \\
\mathbf{2 7 0 n m}\end{array}$ & $\begin{array}{c}\text { meq } \\
\mathbf{f i x} / \mathbf{1 0 0 g} \\
\mathbf{A r g}\end{array}$ & $\begin{array}{c}\text { Rendimento de } \\
\text { intercalação(\%) }\end{array}$ \\
\hline $30^{\circ} \mathrm{C}$ & 0,462 & 12,0 & 18,2 \\
\hline & 0,476 & 10,0 & 15,1 \\
\hline & 0,439 & 15,0 & 22,7 \\
\hline $110^{\circ} \mathrm{C}$ & 0,420 & 17,0 & 25,7 \\
\hline & 0,422 & 17,0 & 25,7 \\
\hline & 0,430 & 16,0 & 24,2 \\
\hline
\end{tabular}

Tabela 3:Influência da concentração de aciclovir no processo

\begin{tabular}{|c|c|c|c|}
\hline $\begin{array}{c}\text { meq/100g } \\
\text { arg }\end{array}$ & $\begin{array}{c}\text { Abs. } \\
\mathbf{2 7 0 n m}\end{array}$ & $\begin{array}{c}\text { meq fix/100g } \\
\text { arg }\end{array}$ & $\begin{array}{c}\text { Rendimento de } \\
\text { intercalação(\%) }\end{array}$ \\
\hline 66 & 0,462 & 12,0 & 18,2 \\
\hline & 0,476 & 10,0 & 15,1 \\
\hline & 0,439 & 15,0 & 22,7 \\
\hline 88 & 0,592 & 17,8 & 20,2 \\
\hline & 0,517 & 27,2 & 30,9 \\
\hline & 0,610 & 15,5 & 17,6 \\
\hline 150 & 0,110 & 59,6 & 39,7 \\
\hline & 0,096 & 77,0 & 51,3 \\
\hline & 0,115 & 36,6 & 24,4 \\
\hline 444 & 0,078 & 171,0 & 38,5 \\
\hline & 0,078 & 171,0 & 38,5 \\
\hline & 0,079 & 176,0 & 39,6 \\
\hline 888 & 0,079 & 159,0 & 17,9 \\
\hline & 0,072 & 154,0 & 28,6 \\
\hline & 0,070 & 278,0 & 31,3 \\
\hline
\end{tabular}

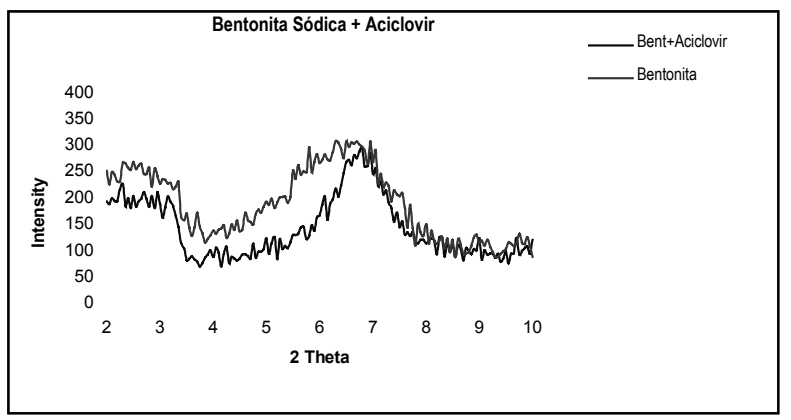

Figura 1: Difratograma da intercalação argila sódica aciclovir em comparação à bentonita sódica isolada. 


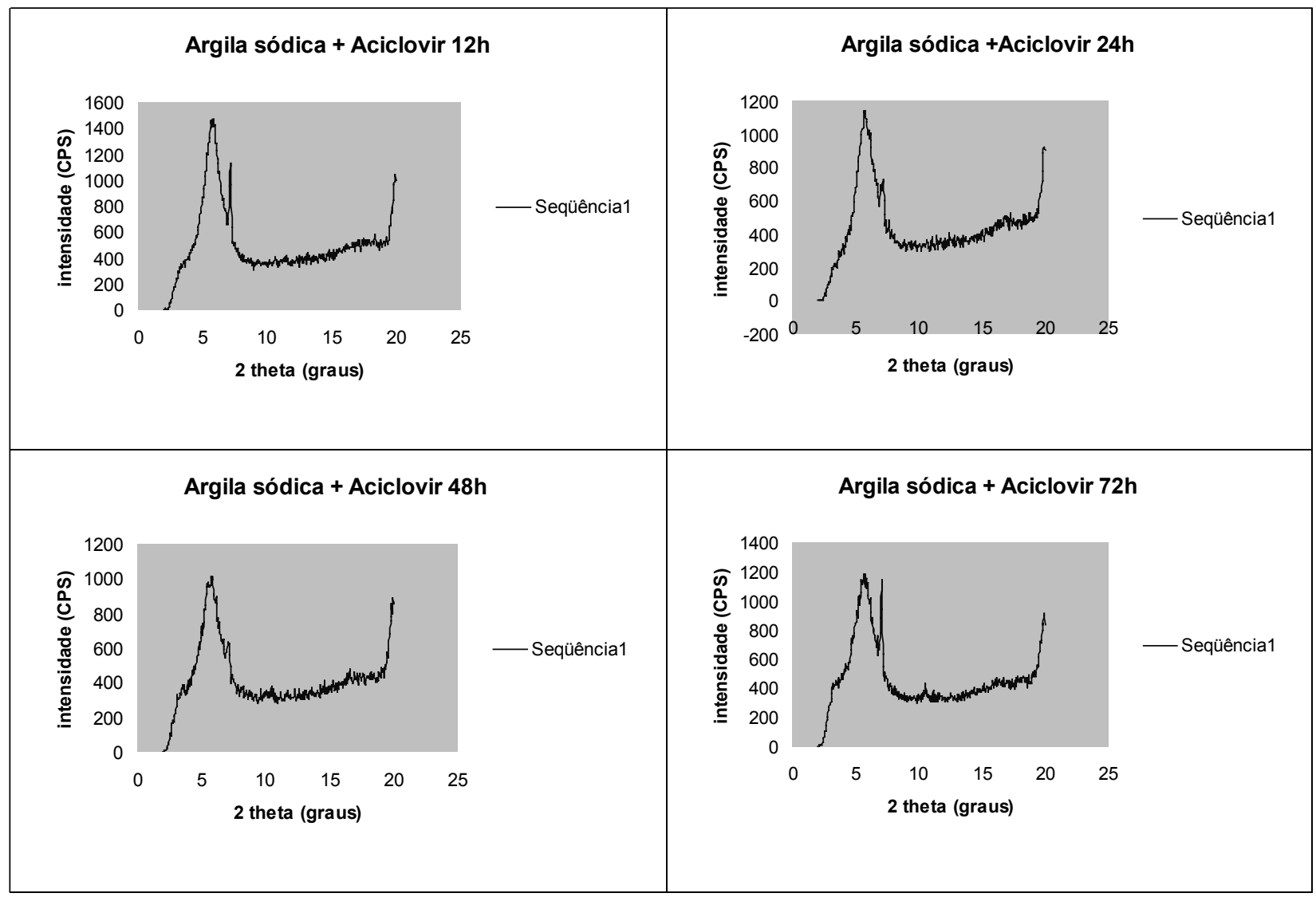

Figura 2: Resultados das reações de intercalação argila sódica aciclovir

Uma redução significativa na quantidade de fármaco intercalada ( $84 \mathrm{meq}$ fix $/ 100 \mathrm{~g}$ arg) foi verificada neste caso como conseqüência da baixa lipofilicidade do mesmo, o que contraria a hipótese inicial de maior afinidade da argila organofílica frente à argila sódica pelo fármaco, visto ser esta muito pouco solúvel em água, sendo o difratograma obtido exibido na Figura 3.

\section{CARACTERIZAÇÃO DOS NANOCOMPÓSITOS ACICLOVIR - ARGILA SÓDICA E ACICLOVIR - ARGILA ORGANOFÍLICA ELEITOS}

De acordo com os espectros de infravermelho (Figuras 4 e 5), não houve qualquer deslocamento significativo dos sinais observados nos materiais de partida, em ambos os casos, de onde conclui-se que a técnica não se mostra efetiva na elucidação da formação de nanocompósitos.

Sobrepondo-se os resultados da difração de raios X das argilas puras com os materiais intercalados (Figuras 6 e 7), pode-se verificar um aumento do espaçamento basal das argilas nos materiais provindo das reações, o que é ratificado com o cálculo destes aumentos $(3,4 \AA$ e $10,3 \AA$ para argila sódica e organofílica, respectivamente), de acordo com a equação de Bragg (Tabela 4).

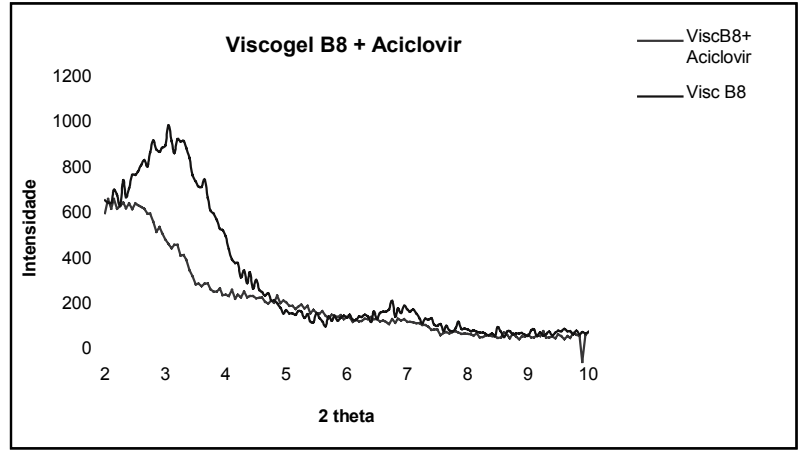

Figura 3: Resultados das reações de intercalação argila sódica aciclovir Resultados das reações de intercalação viscogel B8 - aciclovir 


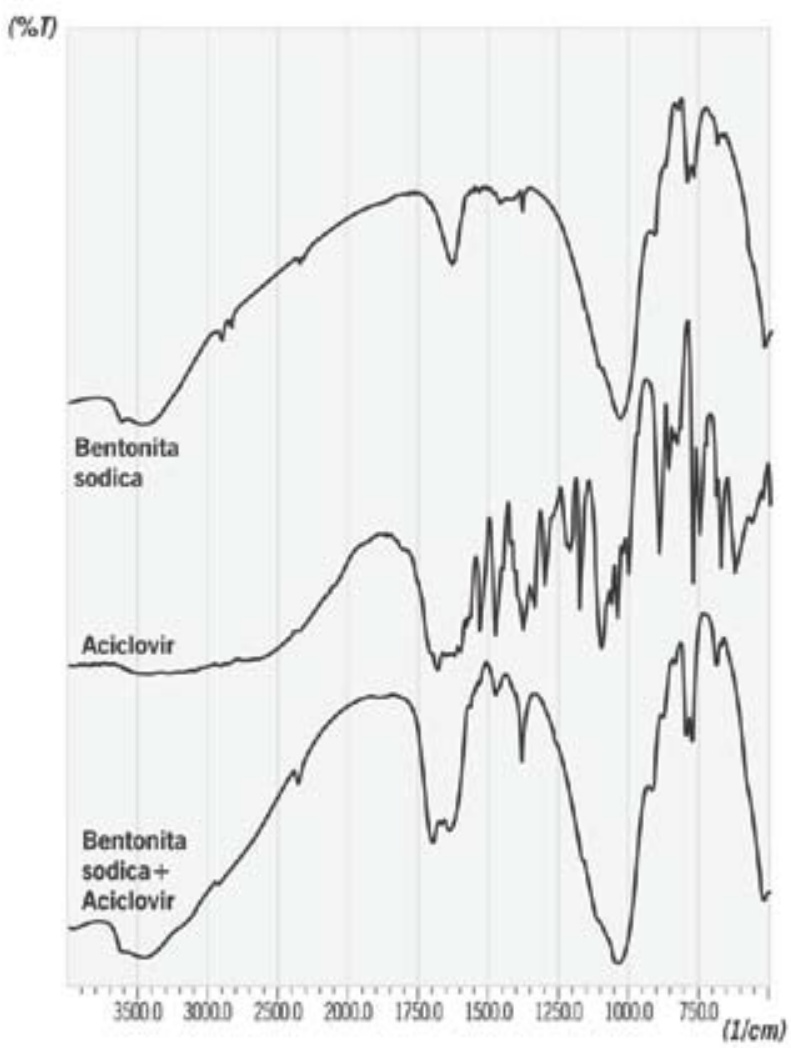

Figura 4: Espectros de infravermelho da bentonita sódica pura, aciclovir e bentonita sódica intercalada com aciclovir

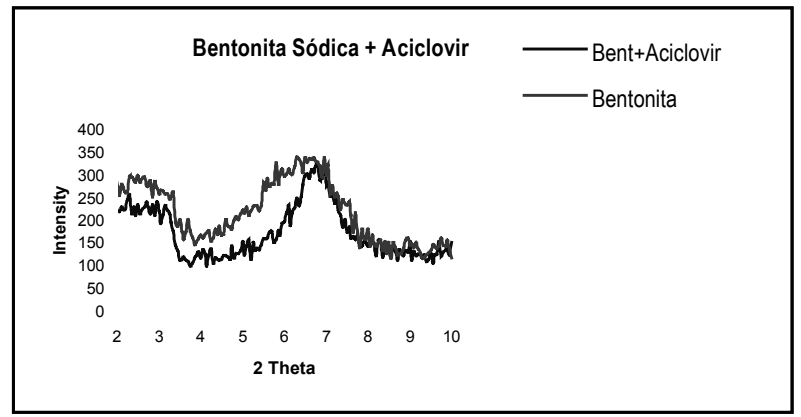

Figura 6: Difratogramas de amostras de bentonita sódica pura e intercalada com aciclovir

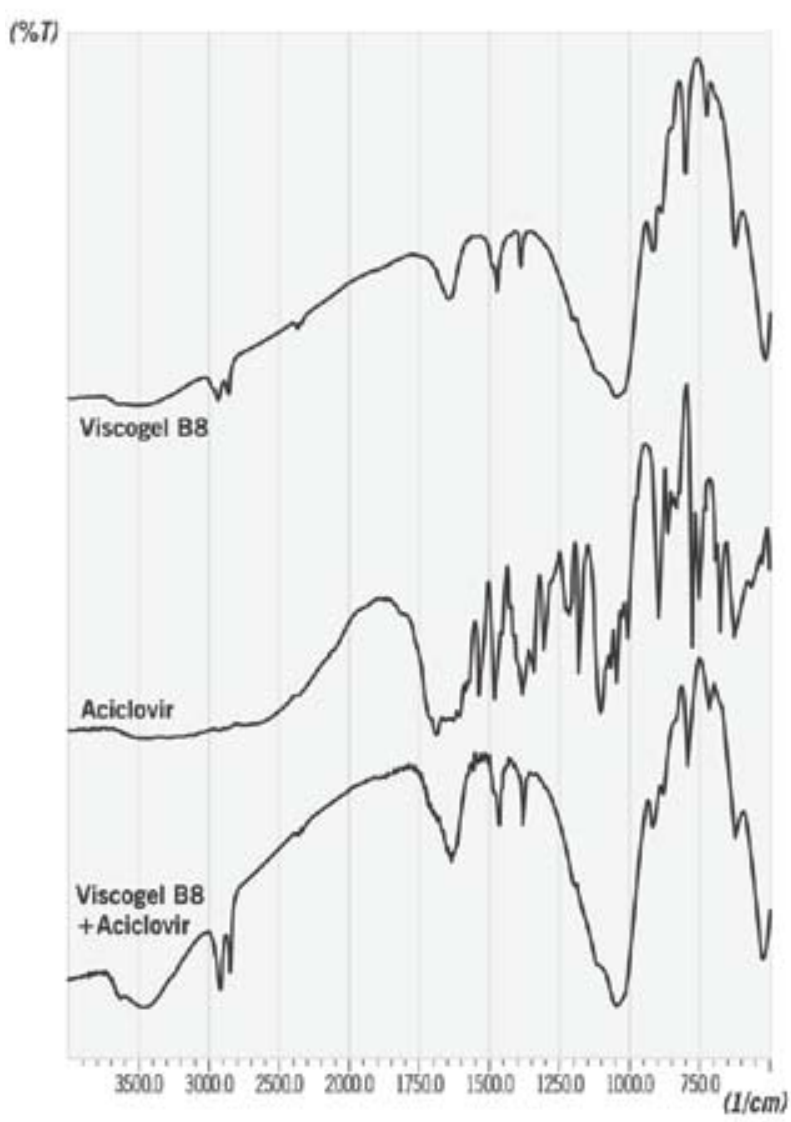

Figura 5: Espectros de infravermelho da bentonita sódica pura, aciclovir e bentonita sódica intercalada com aciclovir

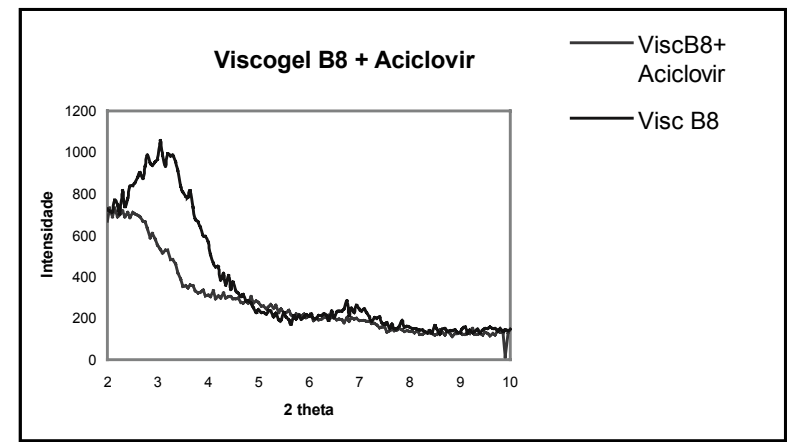

Figura 7: Difratogramas de amostras de viscogel $\mathrm{B} 8^{\circledR}$ puro e intercalado com aciclovir 
Considerando-se as dimensões moleculares do aciclovir e o espaçamento original das argilas estudadas, pode se concluir que o fármaco se inseriu no interior do espaço interlamelar, originando, provavelmente, um nanocompósito intercalado, possivelmente orientado na forma de uma monocamada.

\section{ENSAIOS DE DISSOLUÇÃO}

Os estudos de dissolução realizados com os comprimidos oriundos dos nanocompósitos obtidos demonstraram um evidente retardo na dissolução do aciclovir, com as duas argilas consideradas, como pode ser observado pela Figura 8.

Quando o aciclovir foi intercalado com a bentonita organofílica, apenas $2 \%$ do aciclovir contido na formulação foi liberado para o meio de dissolução, ao passo que uma quantidade próxima a $70 \%$ é liberada na ausência de bentonita. A mistura física destes componentes também promoveu o retardo da liberação do ativo, porém de forma menos eficiente do que quando o ativo foi intercalado (56\% versus $2 \%$ de aciclovir liberado, respectivamente).

A intercalação do ativo com a bentonita sódica também retardou eficientemente a liberação do fármaco para o meio de dissolução, promovendo a liberação de apenas $10 \%$ do ativo durante todo o ensaio. A mistura física destes componentes também gerou um retardo na liberação de aciclovir, porém tênue quando comparada à formulação contendo o nanocompósito $(62 \%$ versus $10 \%$, respectivamente).

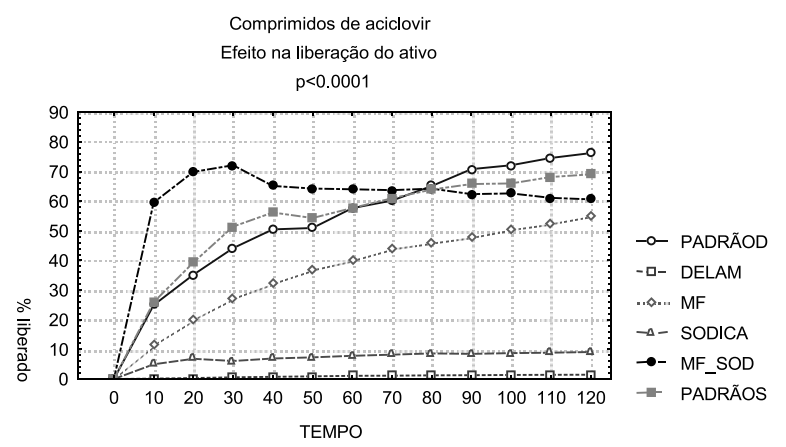

Figura 8: Efeito da bentonita sódica e delaminada na liberação do aciclovir

\section{Conclusões}

Pelos resultados obtidos, mostra-se evidenciada a possibilidade de formular aciclovir em sistemas de liberação modificada, como carreadores nanométricos derivados de silicatos lamelares. O viscogel $B 8^{\circledR}$ se mostrou bastante eficaz em retardar a dissolução do ativo testado, o que se deve, possivelmente, ao caráter lipofílico inerente a esse material, apontando o uso desta argila como uma nova matriz para liberação prolongada de fármacos.

Estudos de estabilidade subseqüentes deverão ser realizados para se obter novos medicamentos com base nos sistemas aqui desenvolvidos.

Tabela 4: Valores de espaçamento basal $\left(\mathrm{d}_{001}\right)$ e variação do espaçamento basal $\left(\Delta \mathrm{d}_{001}\right)$ dos nanocompósitos obtidos

\begin{tabular}{|l|c|c|}
\hline \multicolumn{1}{|c|}{ Amostra } & $\mathbf{d}_{\mathbf{0 0 1}}$ & $\mathbf{\Delta d}_{\mathbf{0 0 1}}$ \\
\hline Bentonita Sódica & $9,5 \AA$ & - \\
\hline Viscogel B8 $\AA$ & $28,95 \AA$ & - \\
\hline Bentonita Sódica + Aciclovir & $12,9 \AA$ & $3,4 \AA$ \\
\hline Viscogel B8 $\AA+$ Aciclovir & $39,25 \AA$ & $10,3 \AA$ \\
\hline
\end{tabular}

\section{REFERÊNCIAS}

1. Afshar, M.; Prescott, C.D.; Varani, G.; Current Opinion in Biotechnology 2001, 10 (1), 59.

2. Lockhart, D. J.; Ho, M. H.; Wodicka, L.; European Patent Application 2004, 418, 244.

3. Albuquerque, U. P.; Hanazaki, N.; Rev. bras. farmacogn. 2006, 16 ,678-689.

4. Hardman J. G.; Limbird, L. E.; Gilman, A. G.; Goodman \& Gilman's The Pharmacological Basis of Therapeutics, 11th ed., 2007.

5. Duran, N., De Azevedo, M.M.M.; Rede de Nanobiotecnologia. UNICAMP, www.comciencia. br, acessado em 01/04/2008. 
6. Farrel, S. Hesketh, R. P. An introduction to drug delivery for Chemical Engineers. em $<$ http:// engineering.eng.rowan.edu/ hesketh/hesketh/ cee\%20drug\%20delivery.pdf Companies envolved in polymeric drug delivery.htm $>$ Acessado em 01/03/2008.

7. Lupi, O.; An. Bras. Dermatol. 2000, 75(3), 261-275.

8. Schaffazick, S. R.; Guterres, S. S.; Freitas, L. L.; Pohlmann, A. R.; Química Nova 2003, 26 (5).

9. Schaffazick, S.R.; Pohlmann, A.R.; Dalla-Costa, T.; Guterres S.S.; European Journal of Pharmaceutics and Biopharmaceutics 2003, 56 (3), 501-505.

10. Prista, L. N.; Alves, A. C.; Morgado, R.; Tecnologia Farmacêutica, 4th ed. Porto: Calouste Gulbenkian, 1995.

11. Lopes, C.M.; Lobo, J.M.S.; Costa, P.; Brazilian Journal of Pharmaceutical Sciences 2005, 41 (2).
12. Moghimi, S. M.; Hunter, A. C.; Murray, J. C.; Pharmacological Reviews 2001, 53 (2), 283-318.

\section{Flávia Almada do Carmo', Lúcio Mendes Cabral*1, Camila Braga Dornelas ${ }^{2}$ e Michele Villardi'}

\footnotetext{
${ }^{1}$ Faculdade de Farmácia, Departamento de Medicamentos, Laboratório de Tecnologia Industrial Farmacêutica, Universidade Federal do Rio de Janeiro.

${ }^{2}$ Instituto de Macromoléculas Professora Eloisa Mano, Universidade Federal do Rio de Janeiro.

*E-mail:1mcabral@pharma.ufrj.br
} 